\title{
ESTUDO TERMODINÂMICO DA INFLUÊNCIA DE MN, NB, S E TI NA PRECIPITAÇÃO EM AÇOS HSLA*
}

\author{
Joelma Krugel Brites ${ }^{1}$ \\ João Batista Ribeiro Martins ${ }^{2}$ \\ Mariane Gonçalves de Miranda ${ }^{3}$ \\ Marcos Tadeu D'Azeredo Orlando ${ }^{4}$ \\ Estéfano Aparecido Vieira ${ }^{5}$
}

\section{Resumo}

Com a perspectiva do uso de aços API em ambientes sour service, o controle de inclusões e precipitados é essencial para bom desempenho. A precipitação de carbonetos e nitretos de $\mathrm{Nb}$ e Ti, assim como a formação do MnS foram analisados criticamente em aços de alta resistência e baixa liga - HSLA- com Mn entre 0,3 e $1,6 \%$, Nb $0,02-0,1 \%$, S $0,001-0,005 \%$ e $\mathrm{Ti} 0-0,02 \%$ em peso. Os valores da temperatura de precipitação do $\mathrm{MnS}, \mathrm{Nb}(\mathrm{C}, \mathrm{N})$ e $\mathrm{Ti}(\mathrm{C}, \mathrm{N})$ foram obtidos por meio do software ThermoCalc ${ }^{\mathrm{TM}}$. A partir desses resultados, possíveis tendências da precipitação foram discutidas e relacionadas com a temperatura de nãorecristalização, traçando um comparativo entre temperaturas de processo e precipitação. Três aços produzidos com a composição estudada atenderam os requisitos mecânicos de tração da norma API 5L X65M.

Palavras-chave: ThermoCalc ${ }^{\mathrm{TM}}$; Precipitação; Aços HSLA.

\section{THERMODYNAMICS STUDY OF THE INFLUENCE FROM MN, NB, S AND TI ON HSLA PRECIPITATION}

\section{Abstract}

Precipitation of $\mathrm{Nb}$ and $\mathrm{Ti}$ carbonites and nitrides, as well as MnS precipitation, were analysed in high strength low alloy steels - HSLA - with Mn between 0,3-1,6\%, Nb $0,02-0,1 \%$, S $0,001-0,005 \%$ and Ti $0-0,02 \%$ wt. ThermoCalc ${ }^{\mathrm{TM}}$ software were used to obtain precipitation temperature from $\mathrm{MnS}, \mathrm{Nb}(\mathrm{C}, \mathrm{N})$ and $\mathrm{Ti}(\mathrm{C}, \mathrm{N})$. From this data, possible precipitation behaviours were considered and related with nonrecrystallization temperature $\left(\mathrm{T}_{\mathrm{nr}}\right)$, and therefore tracking a comparison between process temperature and precipitation. Three steels produced with the studied composition filled the requirements of the API 5L X65M standard

Keywords: ThermoCalc ${ }^{\mathrm{TM}}$; Precipitation; HSLA steels.

\footnotetext{
Mestranda em Eng. Metalúrgica de Materiais, PROPEMM, IFES, Vitória, ES, Brasil.

2 Doutorando em Eng. Metalúrgica - UFF-VR, Pesquisador ArcelorMittal-Global R\&D / Professor IFES, Vitória, ES, Brasil

3 Mestranda em Engenharia Mecânica, PPGEM, UFES, Vitória, ES, Brasil.

4 Doutor em Física, Professor PPGEM, UFES, Vitória, ES, Brasil.

5 Doutor Eng. Materiais, PROPEMM, IFES, Vitória, ES, Brasil..
} 


\section{INTRODUÇÃO}

Os aços de alta resistência e baixa liga (high strenght low alloy - HSLA) são empregados na indústria petrolífera devido a sua resistência mecânica aliada à resistência a corrosão[1]. Com o uso desses aços em ambientes cada vez mais agressivos, o controle de inclusões e precipitados formados é essencial para o bom desempenho do produto em sour service [2]. Para tanto, adiciona-se elementos de liga como $\mathrm{Mn}$, $\mathrm{Nb}$ e $\mathrm{Ti}$ que, embora exerçam diferentes mecanismos nos aços, coletivamente colaboram para o aumento da resistência mecânica dos HSLA.

Dentre os diversos mecanismos que esses elementos desenvolvem, são citados: controle do tamanho de grãos austeníticos no forno de reaquecimento de placas pelo Ti; por meio do Mn, o endurecimento por solução sólida; e por fim o Nb, que exerce endurecimento por precipitação, aumento da temperatura de nãorecristalização $-\mathrm{T}_{\mathrm{nr}}$ - e o refino do grão ferrítico [3,4]. Entretanto, a maximização do desempenho desses mecanismos é dependente da adequação das temperaturas de processo à precipitação. Com esse objetivo, o estudo da termodinâmica se faz necessário para prever o comportamento dos precipitados em função da temperatura.

Por meio do ThermoCalc ${ }^{\mathrm{TM}}$, simulou-se diversas combinações variando os teores de $\mathrm{Mn}, \mathrm{Nb}$, Ti e S. A partir desse levantamento, as temperaturas de formação foram comparadas entre si e com a temperatura de não-recristalização - Tnr, visando estimar os parâmetros ideais de laminação e composição química. Por fim, para comparar as simulações com dados experimentais, ensaios de tração e micrografias foram feitos a fim de estudar o efeito dos elementos de liga no aço.

\section{MATERIAIS E MÉTODOS}

Para obter um parâmetro de comparação da influência dos elementos de liga nas temperaturas de precipitação do $\mathrm{MnS}, \mathrm{Nb}(\mathrm{C}, \mathrm{N})$ e $\mathrm{Ti}(\mathrm{C}, \mathrm{N})$, simulações termodinâmicas foram realizadas no software ThermoCalc ${ }^{\mathrm{TM}}$, permutando $\mathrm{Mn}, \mathrm{Ca}$, $\mathrm{Nb}, \mathrm{S}$ e Ti conforme dado na Tabela 1. A versão utilizada foi a $2017 \mathrm{a}$, com banco de dados TCFE8. Já na Tabela 2 estão descritas as composições dos aços usados no ensaio de tração uniaxial.

Tabela 1 - Composições utilizadas no software ThermoCalc ${ }^{\mathrm{TM}}$

\begin{tabular}{lccccccc}
\hline Elemento & $\mathrm{Ti}$ & $\mathrm{Nb}$ & $\mathrm{Mn}$ & $\mathrm{S}$ & $\mathrm{C}$ & $\mathrm{Si}$ & $\mathrm{Ca}$ \\
\hline$\%$ peso & $0.0-0.2$ & $0.02-0.10$ & $0.3-1.8$ & $0.001-0.003$ & $\leq 0,12$ & $\leq 0,45$ & $0-0,002$ \\
\hline
\end{tabular}

Tabela 2 - Composições químicas dos aços empregados

\begin{tabular}{l|cccccc}
\hline \multicolumn{7}{c}{ Elementos (\% em peso) } \\
\hline Aço & $\mathrm{C}$ & $\mathrm{Mn}$ & $\mathrm{N}$ máximo & $\mathrm{S}$ máximo & $\mathrm{Nb}$ & $\mathrm{Ti}$ \\
$\mathrm{A}$ & 0,05 & 0,40 & 0,0050 & 0,0030 & 0,100 & 0,020 \\
$\mathrm{~B}$ & 0,05 & 1,70 & 0,0050 & 0,0030 & 0,100 & 0,020 \\
$\mathrm{C}$ & 0,05 & 1,70 & 0,0050 & 0,0030 & 0,065 & 0,020 \\
\hline
\end{tabular}

Os ensaios de tração seguem a norma API $5 \mathrm{~L}$, conforme a ISO 6892. A base do corpo media $50,8 \mathrm{~mm}$ de largura, com espessura de chapa $14,00 \mathrm{~mm}$. O corpo de prova foi retirado a $1 / 4$ da largura da bobina na direção transversal de laminação. Foram medidas as propriedades de limite elástico (LE) a $0,5 \%$. A análise por Microscopia Eletrônica de Varredura foi realizada na seção longitudinal, no 
equipamento da marca Jeol de modelo JSM-7100F após polimento com alumina $1 \mu \mathrm{m}$ e ataque com nital $2 \%$.

A equação de $T_{n r}$ foi obtida por Boratto 1988 [5], como mostrado na Equação 1, em que as porcentagens dos elementos estão em fração em massa. Essa equação é válida para o intervalo de: $0.04 \% \leq \mathrm{C} \leq 0.17 \% ; 0.41 \% \leq \mathrm{Mn} \leq 1.90 ; 0.15 \% \leq \mathrm{Si} \leq$ $0.50 \% ; 0.002 \% \leq \mathrm{Al} \leq 0.650 ; \mathrm{Nb} \leq 0.060 \% ; \mathrm{V} \leq 0.120 \% ; \mathrm{Ti} \leq 0.110 \% ; \mathrm{Cr} \leq 0.67 \% ; \mathrm{Ni}$ $\leq 0.45$.

$$
\begin{aligned}
& T_{n r}=887+464[\% C]+6645[\% N b]-644 \sqrt{[\% N b]}+732[\% V]-230 \sqrt{[\% V]} \\
& \quad+890[\% T i]+363[\% A l]-357[\% S i]
\end{aligned}
$$

\section{RESULTADOS E DISCUSSÃO}

\subsection{Temperatura de formação do MnS}

Como esperado, o Mn e S são de grande influência na formação do MnS. Quanto maior o teor desses elementos, maior será a temperatura de formação dessa inclusão de acordo com as simulações obtidas no ThermoCalc ${ }^{\text {TM }}$ (Figura 1). Mesmo com baixo teor de S e Mn haverá potencial termodinâmico para a formação do MnS. A presença do $\mathrm{Ca}$ e $\mathrm{O}$ foi desconsiderada nas simulações, sendo analisada apenas a formação de precipitados a partir do estado sólido da matriz. Caso o Ca fosse considerado, haveria um aumento na temperatura de precipitação da inclusão MnS, como mostra a Figura $2(\mathrm{Ca}=20 \mathrm{ppm})$.
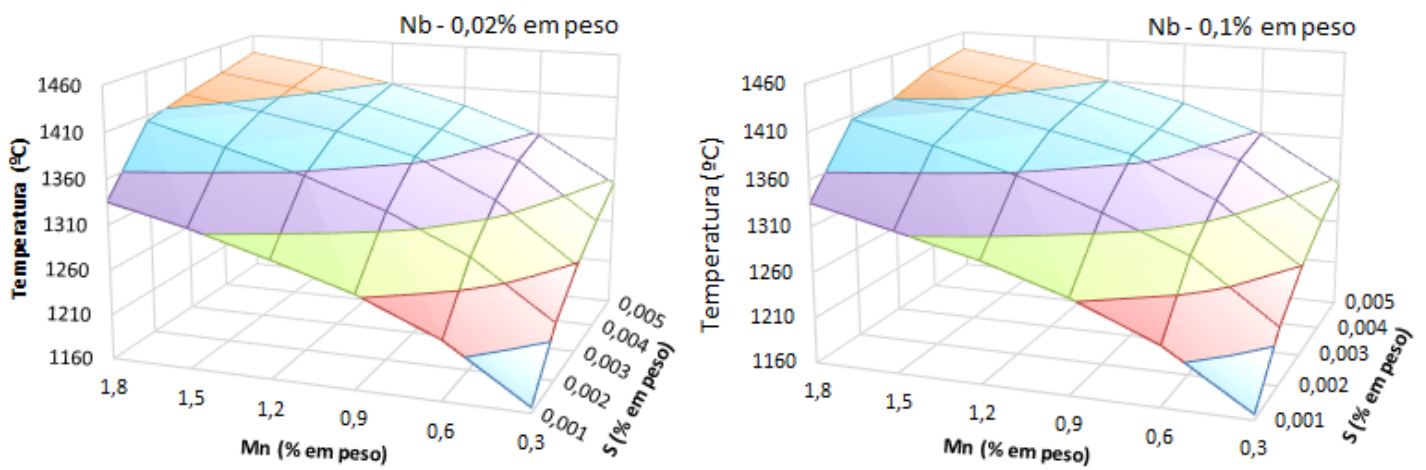

$\square 1410-1460$

$\square 1360-1410$

$\square 1310-1360$

$\square$ 1260-1310

$\square 1210-1260$

$\square 1160-1210$

Figura 1 - Formação do MnS com a) $\mathrm{Nb}=0,02 \%$ e b) $\mathrm{Nb}=0,1 \%$, ambos com $\mathrm{Ti}=0,02 \%$ em peso.

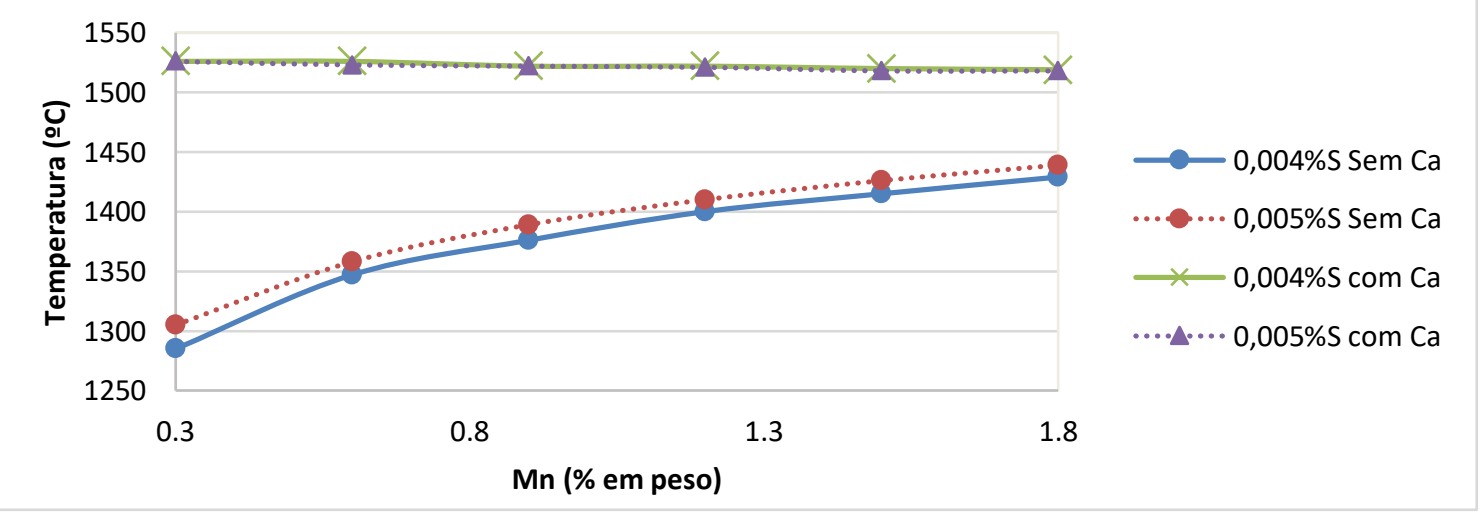

Figura 2 - Temperatura de formação do MnS ao considerar a presença de Ca 
O software considera uma fase única MnS composta de (Ca,Mn)S, cuja temperatura de formação aumenta na presença de $\mathrm{Ca}$, sendo irrelevante para os demais precipitados não-sulfurosos como $\mathrm{Ti}(\mathrm{C}, \mathrm{N})$ e $\mathrm{Nb}(\mathrm{C}, \mathrm{N})$. Caso o $\mathrm{O}$ também seja considerado, a temperatura de formação do (Ca,Mn)S reduziria devido à concomitante redução do Ca disponível. Desta forma, o (Ca, Mn)S possui a maior temperatura de formação, seguida do (Ca,Mn)S com O disponível na liga e MnS sem Ca e O.

A partir dessas informações, observou-se que a temperatura de formação para a maioria das composições está acima da utilizada em forno de reaquecimento de placas $\left(\mathrm{T}>1220^{\circ} \mathrm{C}\right)$. Desta forma, para teores de $\mathrm{Mn}$ acima de $0,9 \%$ em peso e 30 ppm de $\mathrm{S}$, a ressolubilização do MnS será nula ou baixa após a solidificação, estando essa inclusão não globulizada. É importante ressaltar que ainda são necessárias maiores informações sobre a cinética de dissolução do $\mathrm{MnS} /(\mathrm{Ca}, \mathrm{Mn}) \mathrm{S}$, para comprovar se essa é ágil suficiente para dissolver o MnS na temperatura de reaquecimento de placas.

Pela Figura 3, para baixo teor de $S$ (10 ppm), o Ti passa a ser influente apenas acima de $0,03 \%$. Isso ocorre devido à formação do $\mathrm{Ti}_{4} \mathrm{~S}_{2} \mathrm{C}_{2}$, o qual também é responsável pelo declínio da curva, cujo valor de S é 50 ppm. Tendo em vista que a formação desse carbossulfeto decorre apenas para $S>30$ ppm ou $\mathrm{Ti}>0,02 \% \mathrm{em}$ peso ele foi desconsiderado nesse estudo.

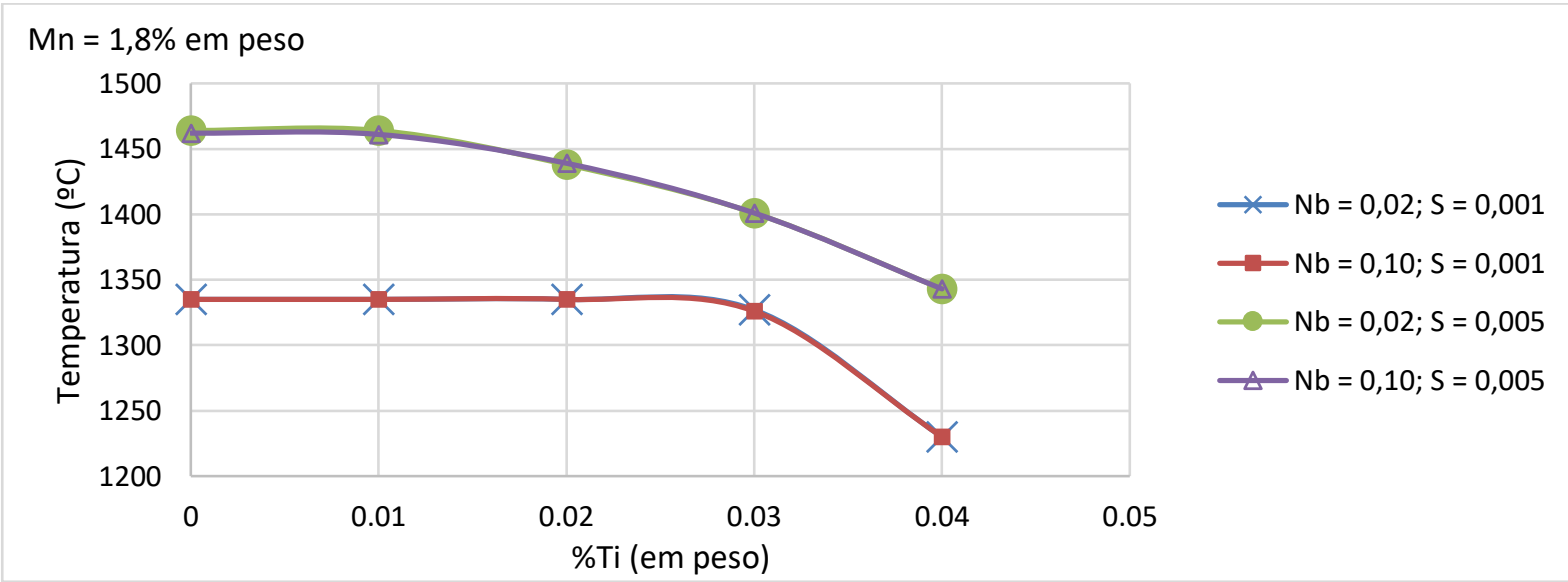

Figura 3 - Curva de precipitação para o Mn

\subsection{Temperatura do $\mathrm{Ti}(\mathrm{C}, \mathrm{N})$}

Os elementos de maior impacto na formação do TiN são o $\mathrm{Mn}$ e Ti. Para baixos teores de $\mathrm{Ti}(<0,02 \%)$, a fase $\mathrm{Ti}(\mathrm{C}, \mathrm{N})$ apresenta menor estabilidade, precipitando em menores temperaturas (Figura 4). Embora o TiN seja estável a altas temperaturas $\left(\mathrm{T}>1250^{\circ} \mathrm{C}\right)$, observa-se que o $\mathrm{Mn}$ possui influência significativa sobre sua temperatura de formação, reduzindo-a em mais de $30^{\circ} \mathrm{C}$, por exemplo, quando $\mathrm{Ti}=$ 0,01\% (Figura 5). 

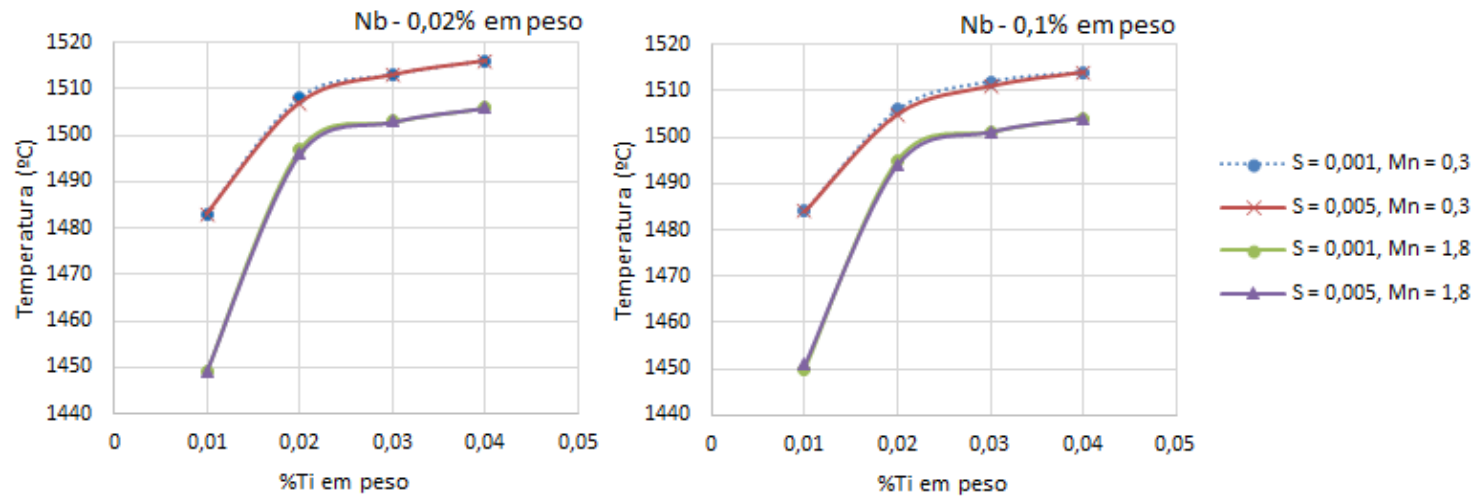

Figura 4 - Formação do Ti(C,N) com a) $0,02 \% \mathrm{Nb}$ e b) $0,1 \% \mathrm{Nb}$
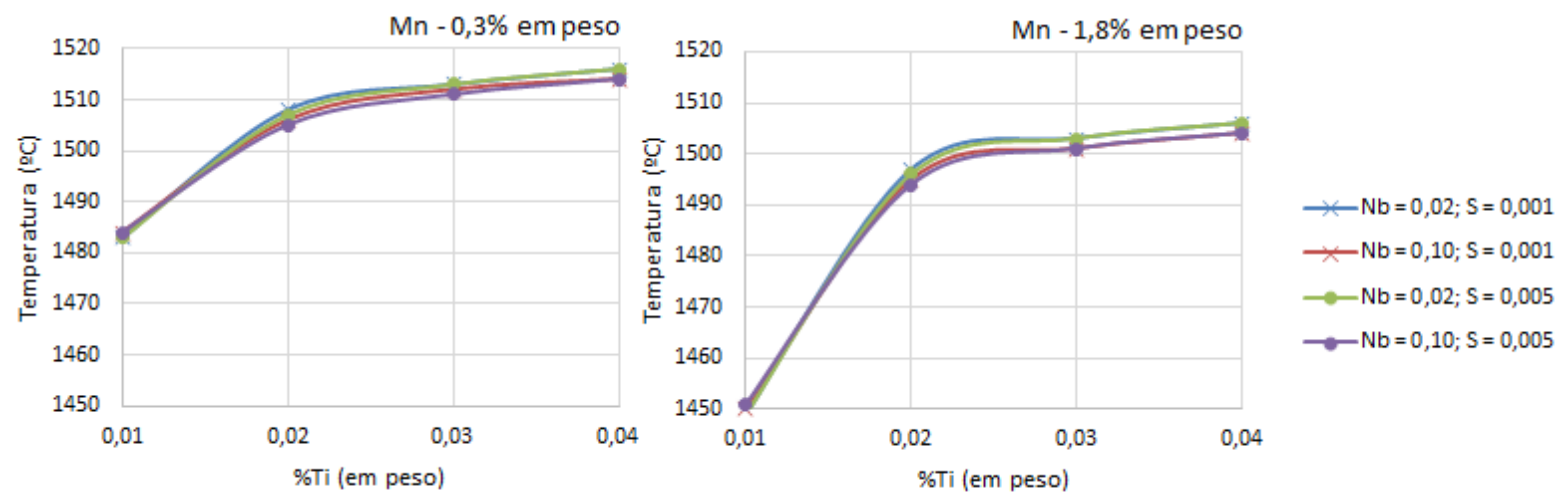

Figura 5 - Formação do $\mathrm{Ti}(\mathrm{C}, \mathrm{N}) \mathrm{com}$ a) $\mathrm{Mn}=0,3 \%$ e b) $\mathrm{Mn}=1,8 \%$ em peso

Apesar de se encontrar $\mathrm{Nb}$ dissolvido no $\mathrm{Ti}(\mathrm{C}, \mathrm{N})$, sua atividade não interfere de forma representativa na formação desse precipitado.

É observado também que esse carbonitreto de Ti é estável acima da temperatura de reaquecimento de placas, portanto a ressolubilização é inviabilizada.

\subsection{Temperatura de formação do $\mathrm{Nb}(\mathrm{C}, \mathrm{N})$ e a $\mathrm{T}_{\mathrm{nr}}$}

A Figura 6 mostra a capacidade de estabilização do $\mathrm{Nb}$ no seu carbonitreto, mantendo-se $\mathrm{Ti}$ constante. Ao variar o teor de $\mathrm{Nb}$ de $0,02 \%$ a 0,10\%, é possível aumentar a temperatura de formação do $\mathrm{Nb}(\mathrm{C}, \mathrm{N})$ em mais de $150^{\circ}$.Observa-se também que, tanto o $\mathrm{Mn}$ quanto o $\mathrm{S}$ são de baixa relevância na temperatura de formação desse precipitado.
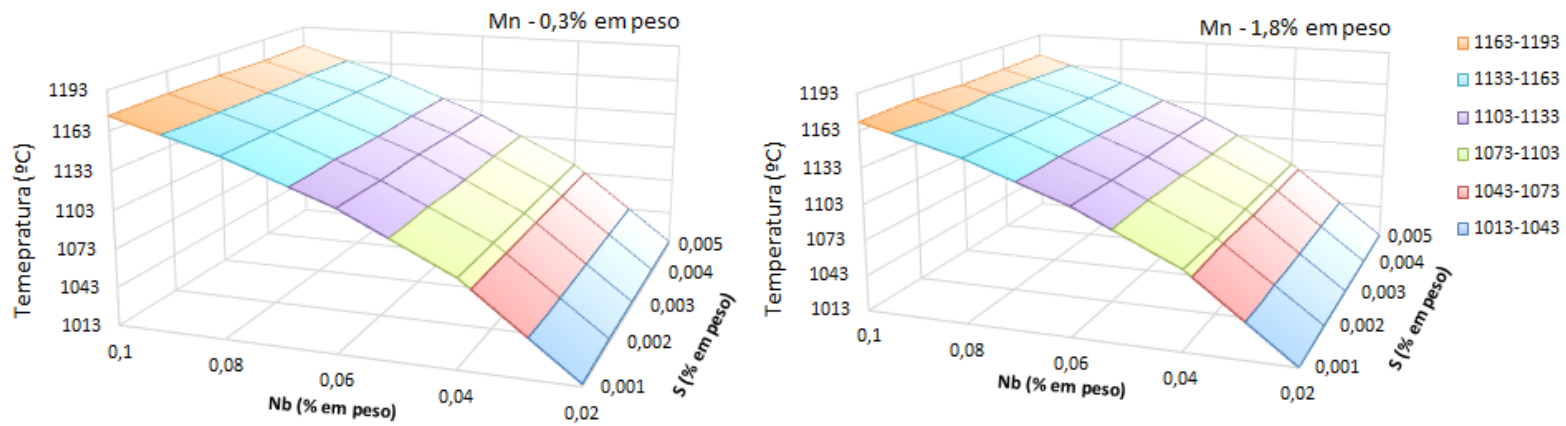

Figura 6 - Temperaturas de formação do $\mathrm{Nb}(\mathrm{C}, \mathrm{N})$ a) $\mathrm{Mn}=0,3 \%$ e b) $\mathrm{Mn}=1,8 \%$ em peso

Outro elemento relevante na formação do $\mathrm{Nb}(\mathrm{C}, \mathrm{N})$ é o Ti. Diversos autores relatam o precipitado como rico em $\mathrm{Nb}$, por conter também $\mathrm{Ti}, \mathrm{C}$ e $\mathrm{N}[1,6,7]$. Isso é possível 
pois o $\mathrm{TiN}$ e $\mathrm{Nb}(\mathrm{C}, \mathrm{N})$ possuem estrutura cristalina idêntica CFC. Como pode ser visto na Figura 7, para teores constantes de $\mathrm{Nb}$ o Ti é capaz de reduzir a temperatura de formação do precipitado, alcançando um valor mínimo a $\mathrm{Ti}=0,02 \%$.
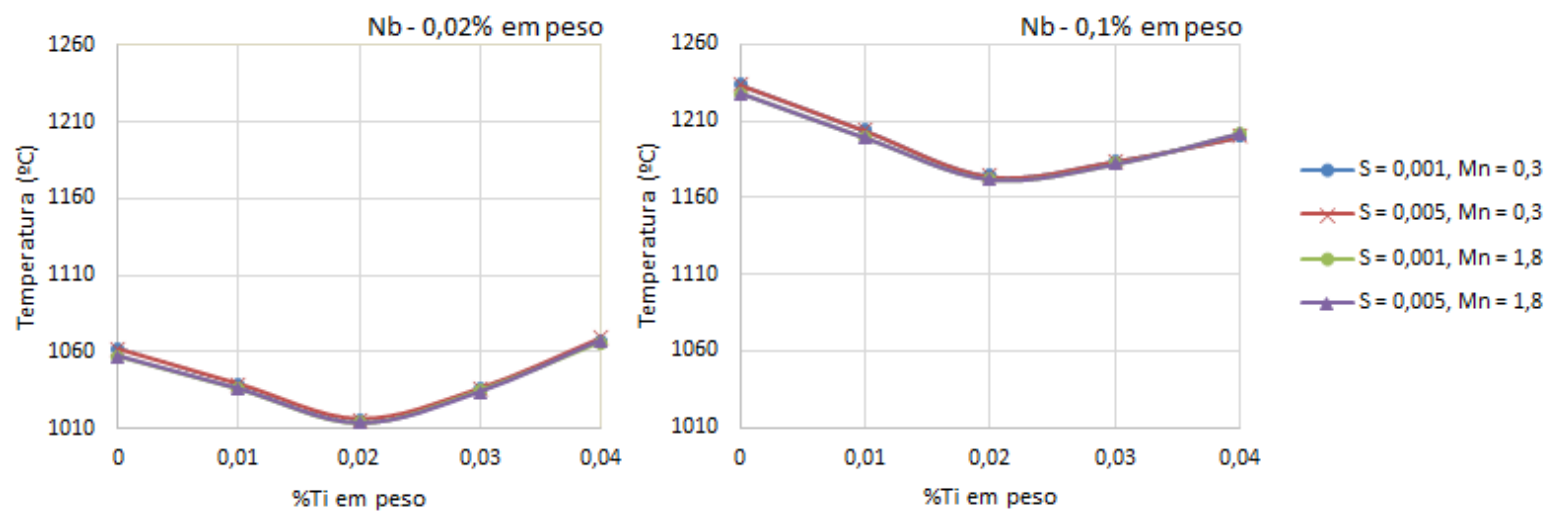

Figura 7 - Temperaturas de formação do $\mathrm{Nb}(\mathrm{C}, \mathrm{N})$ a) $\mathrm{Nb}=0,02 \%$ e b) $\mathrm{Nb}=0,1 \%$ em peso

Tomando a composição $\mathrm{Nb}=0,02 \%, \mathrm{Mn}=0,3 \%, \mathrm{~N}=0,006 \%$ e $\mathrm{S}=0,001 \%$ em peso, obteve-se a variação da composição inicial do $\mathrm{Nb}(\mathrm{C}, \mathrm{N})$ na Figura 8 , com a temperatura de precipitação em pontilhado.

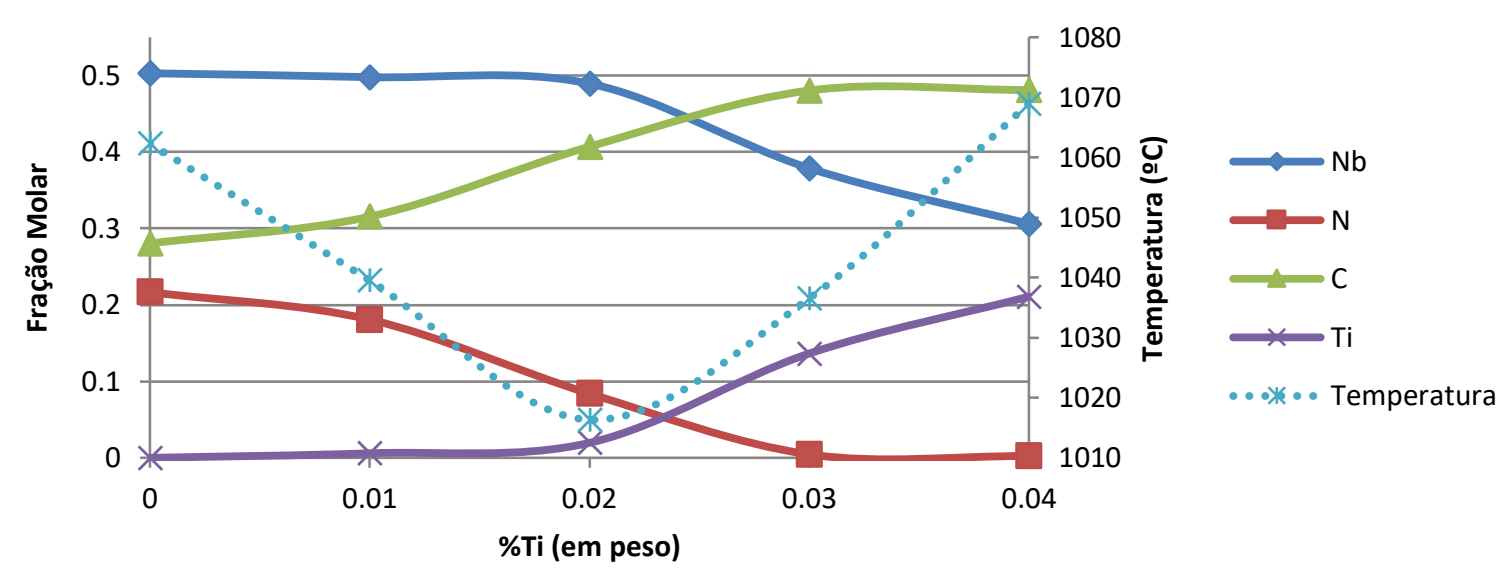

Figura 8 - Composição molar da fase $\mathrm{Nb}(\mathrm{C}, \mathrm{N})$, com a temperatura de precipitação em pontilhado

Como visto na Figura 8, quanto maior a fração de $\mathrm{N}$ e Ti inicial, maior é temperatura de precipitação do complexo de $\mathrm{Nb}$. Baseado nos resultados de Craven et al, 2000 [7], primeiramente é formado o $\mathrm{Ti}(\mathrm{C}, \mathrm{N})$ e apenas o $\mathrm{N}$ restante estará disponível pra formar o $\mathrm{Nb}(\mathrm{C}, \mathrm{N})$. Ao aumentar o teor de $\mathrm{Ti}$ até aproximadamente $0,03 \%$ em peso, todo o $\mathrm{N}$ estaria preferencialmente ligado ao $\mathrm{Ti}$ na temperatura de formação do $\mathrm{Nb}(\mathrm{C}, \mathrm{N})$. Prosseguindo com o aumento de $\mathrm{Ti}$, esse estaria em excesso, tornando-se assim (Nb, Ti)C.

$\mathrm{O}$ aumento da temperatura de formação do $\mathrm{Nb}(\mathrm{C}, \mathrm{N})$, quando esse é rico em $\mathrm{N}$ $(\mathrm{Ti}<0,02 \%)$, é devido à maior estabilidade dos nitretos comparados aos respectivos carbonetos de $\mathrm{Nb} / \mathrm{Ti}$. O Ti também estabiliza esse complexo de $\mathrm{Nb}(\mathrm{C}, \mathrm{N})$. Desta forma, conclui-se que, para a redução da temperatura de formação, termodinamicamente é mais recomendado o teor de $0,02 \%$ de $\mathrm{Ti}$ para $0,006 \% \mathrm{em}$ peso de $\mathrm{N}$.

Dentre as diversas funções do $\mathrm{Nb}$ para laminados a quente, cita-se aumentar a temperatura de não-recristalização $\left(T_{n r}\right)$ e endurecer por precipitação $[4,8]$. Para tais 
condições, deseja-se o retardo da recristalização durante o acabamento e que, concomitantemente, a precipitação ocorra entre os passes. Desta forma, quanto menor a $T_{\text {precipitação }} \mathrm{Nb}(\mathrm{C}, \mathrm{N})$ e maior a $\mathrm{Tnr}$, maior a garantia de que haverá precipitação máxima induzida por deformação. Por consequência, a melhor composição baseada no equilíbrio é de $\mathrm{Ti}=0,02 \%$ para $\mathrm{N}=0,006 \%$ conforme Figura 9. Como a equação da $T_{n r}$ foi extrapolada para o uso nesse estudo, são necessários estudos adicionais para $\mathrm{Nb}>0,06 \%$ a fim de se saber se, de fato, a $\mathrm{T}_{\mathrm{nr}}$ possui tal comportamento em alto $\mathrm{Nb}$. A temperatura de transformação austenita-ferrita no equilíbrio é superior à do resfriamento, portanto haveria tempo suficiente para a nãorecristalização da austenita antes da transformação de fase.
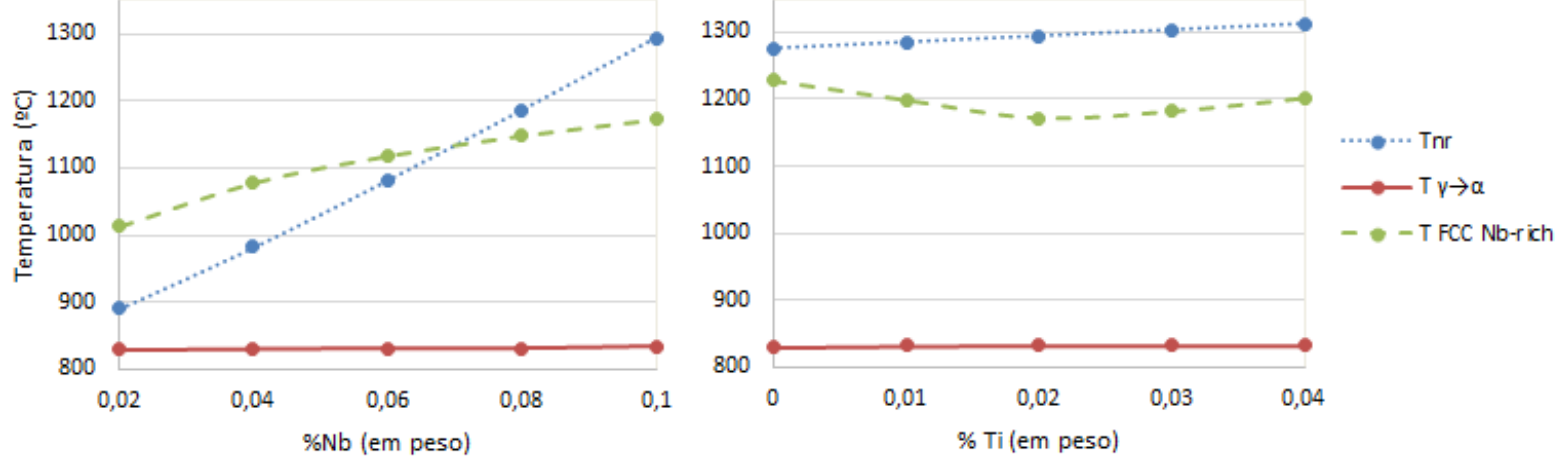

Figura 9 - Temperaturas de precipitação do $\mathrm{Nb}(\mathrm{C}, \mathrm{N}), \mathrm{T}_{\mathrm{nr}}$ e $\mathrm{A}_{\mathrm{e} 3}$, variando a) $\mathrm{Nb}$ e b) $\mathrm{Ti}$

\section{4- Ensaios mecânicos e micrografia}

Os resultados de ensaios mecânicos e do tamanho de grão são apresentados na Tabela 3, e a partir dela conclui-se que a norma API 5L é atendida. É importante frisar que essa norma assegura o produto já conformado, portanto os aços estudados não sofreram o Efeito Bauschinger embora ainda sirvam de parâmetro de comparação.

O Efeito Bauschinger é a diminuição do limite de resistência mecânica devido à aplicação de uma tensão em sentido oposto ao previamente conformado, como ao aplainar um corpo de prova retirado de um tubo, por exemplo [5]. A razão entre o limite de escoamento (LE) e o limite de resistência (LR) é menor que 0,90, o que está abaixo do que a norma estabelece como máximo de 0,93.

Tabela 3 - Resultados dos ensaios de tração uniaxial

\begin{tabular}{lcccccc}
\hline Amostra & Direção & $\begin{array}{c}\mathrm{LE} \\
(\mathrm{MPa})\end{array}$ & $\begin{array}{c}\mathrm{LR} \\
(\mathrm{MPa})\end{array}$ & $\begin{array}{c}\mathrm{LE} / \mathrm{LR} \\
\begin{array}{c}\text { Alongamento } \\
\text { Total }(\%)\end{array}\end{array}$ & $\begin{array}{c}\text { Redução } \\
\text { Área (\%) }\end{array}$ \\
\hline A & $\mathrm{L}$ & $523 \pm 6$ & $606 \pm 5$ & 80 & $40 \pm 1$ & $76 \pm 1$ \\
\hline B & $\mathrm{L}$ & $572 \pm 5$ & $641 \pm 6$ & 89 & $37 \pm 1$ & $75 \pm 1$ \\
\hline C & $\mathrm{L}$ & $521 \pm 6$ & $641 \pm 4$ & 83 & $37 \pm 1$ & $73 \pm 1$ \\
\hline \multirow{2}{*}{ Norma } & Mínimo & 450 & 535 & & & \\
\cline { 2 - 7 } & Máximo & 600 & 760 & 0,93 & & \\
\hline
\end{tabular}

Com maior teor de $\mathrm{Nb}$ e $\mathrm{Mn}$ (amostra B) a resistência mecânica é aumentada. Desconsiderando a influência de outros elementos, a diferença no limite de escoamento entre A e B é proporcional ao teórico, o qual, para cada $1 \%$ em peso de 
Mn pode ocorrer o aumento de $32 \mathrm{MPa}$ na ferrita [4]. Já entre B e C a queda do LE pode ser devido à redução do Nb contido na liga.

Pela Figura 10 é visto que foi obtida estrutura ferrítica em todos os aços. Entretanto, na amostra $A$ há um alto grau de acicularidade e de carboneto de Fe. Já a composição B apresenta ferrita poligonal e $C$ poligonal e acicular, com uma pequena fração de perlita.

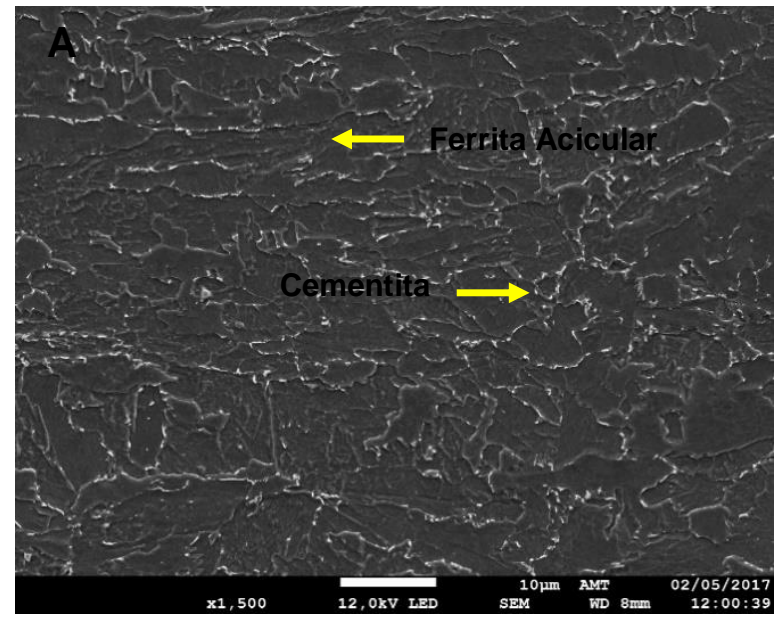

(a)

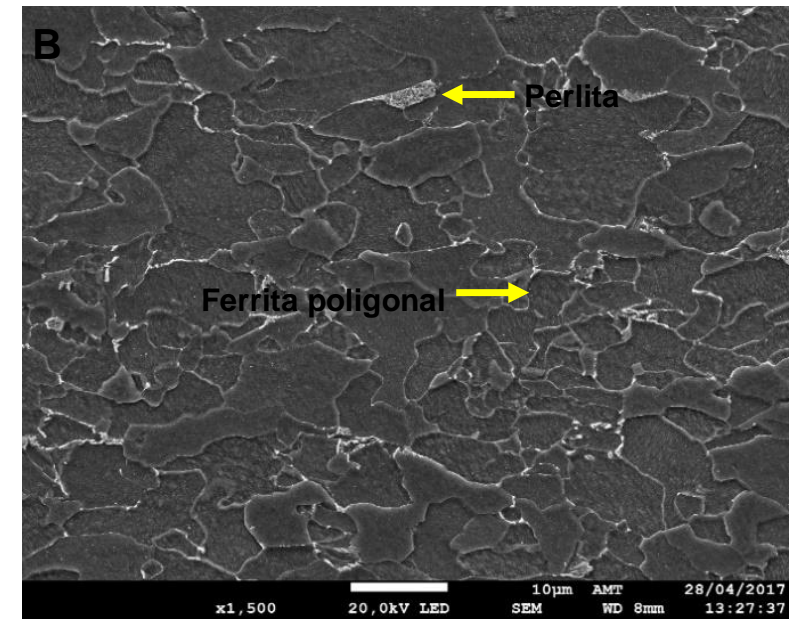

(b)

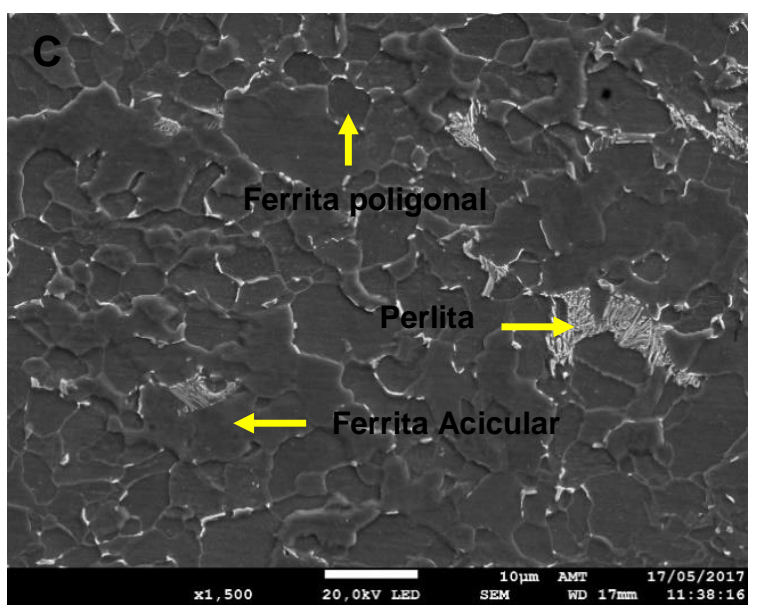

(c)

Figura 10 - Micrografias obtidas no MEV, com ataque Nital 2\%, indicando: (a) aço A com 100\% de ferrita acicular, (b) aço B, ferrita poligonal e (c) aço C, com ferrita poligonal e acicular, além de uma pequena fração volumétrica de perlita.

A partir da comparação entre precipitação termodinâmica, ensaios mecânicos e micrografia, a combinação de alto $\mathrm{Mn}$ e $\mathrm{Nb}$ igual a $0,1 \%$ (B) resultou em microestrutura mais homogênea e efeito cumulativo no aumento da resistência mecânica. São necessárias análises posteriores por microscopia de transmissão para correlacionar a presença e o tamanho de precipitados formados com as propriedades mecânicas.

\section{CONCLUSÃo}


Por meio desse estudo, pode concluir-se que, ao manter constante o N, C, Si, Cr e $\mathrm{Ni}$ :

- O MnS é proporcional com o teor de Mn e S da liga, com pouca influência do teor de $\mathrm{Nb}$ nessa inclusão. Mesmo que haja baixo teor de $\mathrm{Mn}$ e $\mathrm{S}$, há condição termodinâmica para a formação do MnS, que poderá ser solubilizado no forno de reaquecimento de placa para $\mathrm{Mn}>0,9 \%$ e $\mathrm{S}>0,003 \%$ em peso. A presença de $\mathrm{Ca}$ aumenta a temperatura de formação dessa inclusão; Já o O, combinado com o cálcio, reduz a temperatura do (Mn,Ca)S devido à redução do Ca disponível para reação dessa inclusão;

- O Ti $(C, N)$ é influenciado pela presença de Ti e Mn, principalmente quando há razão sub-estequiométrica de Ti. Porém as temperaturas de formação estão acima daquela de reaquecimento de placas ( $\left.T_{\text {precipitação }} 1220^{\circ} \mathrm{C}\right)$. Portanto é esperado baixa ressolubilização nesse processo;

- Já o $\mathrm{Nb}(\mathrm{C}, \mathrm{N})$ é dependente do $\mathrm{Nb}$ e Ti. Quanto maior a porcentagem de $\mathrm{Nb}$ na liga, maior a temperatura de formação. Ao variar o Ti, há uma queda na temperatura de formação quando o Ti alcança aproximadamente relação 1:1. Isso se deve à baixa fração de $\mathrm{N}$ e Ti remanescente para o estabilizar. Portanto, para que se precipite $\mathrm{Nb}(\mathrm{C}, \mathrm{N})$ em menores temperaturas é recomendável manter a relação Ti: $\mathrm{N}$ aproximadamente estequiométrica;

- Para relacionar a temperatura de precipitação do $\mathrm{Nb}(\mathrm{C}, \mathrm{N})$ e a $\mathrm{Tnr}$, é possível a análise somente até $\mathrm{Nb}=0,065 \%$, sendo esse o limite de validade de equação. Ainda assim é possível ver que, quanto maior o teor de $\mathrm{Nb}$, maior é a Tnr e a temperatura de precipitação, então maior será a precipitação de $\mathrm{Nb}(\mathrm{C}, \mathrm{N})$ induzida por deformação;

- As amostras atenderam a norma API 5L na condição de as-rolled, e com alto $\mathrm{Nb}$ e $\mathrm{Mn}$, o aumento da resistência mecânica foi cumulativa pela adição desses elementos. Em todas as amostras há a estrutura ferrítica: A possui alto grau de acicularidade e de carboneto de Fe; B é poligonal; e C poligonal e acicular, com uma pequena fração de perlita. São necessárias análises posteriores no microscópio de transmissão para correlação entre tamanho de precipitado e as temperaturas de precipitação, não sendo possível por análise via MEV.

\section{Agradecimentos}

Os autores agradecem à ArcelorMittal Global R\&D Brazil pelo uso do software ThermoCalc $^{\mathrm{TM}}$ e caracterização do material, à FACTO, ao Ifes e à Ufes pela estrutura fornecida.

\section{REFERÊNCIAS}

1 Gladman T. The Physical Metallurgy of Microalloyed Steels. London: The Institute of Materials; 1997. p 1-260.

2 Gray JM. Low Manganese Sour Service Linepipe Steel. In: Proceedings of the Microalloyed Steels for Sour Service International Seminar. São Paulo: [s.n.]. 2012.

3 Morrison WB. Overview of Microalloying in Steel. In: The Proceeding of The Vanitec Symposium. Guilin: [s.n]. 2000. p.25-35

4 Nishioka K, Ichikawa K. Progress in thermomechanical control of steel plates and their commercialization. Science and Technology of Advanced Materials, 2012;13,. p 1-20. 
5 Llewellyn DT, Hudd RC. Steels: Metallurgy and Applications. 3․ ed. Oxford:

Butterworth-Heinemann; 2000. p 137-198.

6 Boratto F, Barbosa R, Yueand S, Jonas JJ. Effect of Chemical Composition on Critical Temperatures of Microalloyed Steels. THERMEC-88, Vol. 1, ed. by I. Tamura, ISIJ, Tokyo, 1988. 383

7 Craven, AJ. et al. Complex Heterogeneous Precipitation in Titanium - Niobium Microalloyed Al-Killed HSLA Steels - I. (Ti, Nb)(C, N) Particles. Acta Materialia. 2000,48. 3857-3868.

8 Hong SG, Kang KB, Park, CG. Strain-Induced Precipitation of $\mathrm{NbC}$ in $\mathrm{Nb}$ and $\mathrm{Nb}-\mathrm{Ti}$ Microalloyed HSLA Steels. Scripta Materialia. 2002,46. p 163-168.

9 Nishioka K, Ichikawa K. Progress in thermomechanical control of steel plates and their 10 commercialization. Science and Technology of Advanced Materials. 2012,13. p 1-20 